This is a self-archived version of an original article. This version may differ from the original in pagination and typographic details.

Author(s): $\begin{aligned} & \text { Chernyshev, B.A.; Demyanova, A.S.; Goncharov, S.A.; Gurov, Yu. B.; Lapushkin, S. } \\ & \text { V.; Ogloblin, A. A.; Sandukovsky, V. G.; Trzaska, W. H. }\end{aligned}$

Title: The Neutron Structure of the Ground State of $7 \mathrm{He}$

Year: 2019

Version: Accepted version (Final draft)

Copyright: (c) Pleiades Publishing, Inc., 2019

Rights: In Copyright

Rights url: http://rightsstatements.org/page/InC/1.0/?language=en

Please cite the original version:

Chernyshev, B.A., Demyanova, A.S., Goncharov, S.A., Gurov, Y. B., Lapushkin, S. V., Ogloblin, A.A., Sandukovsky, V. G., \& Trzaska, W. H. (2019). The Neutron Structure of the Ground State of 7He. JETP Letters, 110(2), 97-101. https://doi.org/10.1134/s0021364019140030 


\title{
The neutron structure of the ground state of ${ }^{7} \mathrm{He}$
}

\author{
B. A. Chernyshev ${ }^{+1)}$, A.S.Demyanova*, S. A. Goncharov ${ }^{\times}$, Yu. B. Gurov ${ }^{+}$, S. V. Lapushkin ${ }^{+}$, A. A. Ogloblin*, \\ V. G. Sandukovsky ${ }^{\circ}$, W. H. Trzaska ${ }^{\nabla}$ \\ + National Research Nuclear University MEPhI, 115409 Moscow, Russia \\ *National Research Center "Kurchatov Instirtute", 123182 Moscow, Russia \\ ${ }^{\times}$Faculty of Physics, M. V. Lomonosov Moscow State University, 119991 Moscow, Russia \\ ${ }^{\circ}$ Joint Institute for Nuclear Research, 141980 Dubna, Russia \\ $\nabla$ Department of Physics, University of Jyvaskyla, FIN-40014 Jyvaskyla, Finland \\ Submitted 25 December 2018 \\ Resubmitted 27 May 2019 \\ Accepted 27 May 2019
}

DOI: $10.1134 /$ S0370274X19140029

A study of the neutron structure of the ground state of ${ }^{7} \mathrm{He}$ has been performed by means of registration and analysis of the decay channels of the residual nuclei following absorption of stopped pions. The discovery of neutron halos [1] was one of the most important achievements of nuclear physics at the end of the $20^{\text {th }}$ century. It has been traditionally assumed that only bound neutrons may form a halo. Consequently, halo appearances in continuum are not being considered. An indication of a possible existence of a halo-like structure in ${ }^{7} \mathrm{He}$ is provided by the neighboring isotopes of ${ }^{6} \mathrm{He}$ and ${ }^{8} \mathrm{He}$ in which such structures are pronounced. It is natural to expect that the three neutrons surrounding the ${ }^{4} \mathrm{He}$ core in ${ }^{7} \mathrm{He}$ are similar to the two and four neutrons surrounding the ${ }^{4} \mathrm{He}$ core in ${ }^{6} \mathrm{He}$ and ${ }^{8} \mathrm{He}$, respectively. However, the very fact that the valence neutron is located in continuum, may change the structure of ${ }^{7} \mathrm{He}$.

In this paper we propose a novel method of identifying the most important configurations in ${ }^{7} \mathrm{He}$ by utilizing nuclear absorption of stopped pions. We assume that ${ }^{7} \mathrm{He}$ is formed from particles emitted after pion absorption. In this case the non-resonant part of the spectrum of the emitted particles would reflect their contribution to the formation of ${ }^{7} \mathrm{He}$. If only one configuration were to play a noticeable role, it would be expected that this configuration would also determine the type of the halolike configuration in ${ }^{7} \mathrm{He}$. Here we concentrate on the decays resulting from the ${ }^{9} \mathrm{Be}\left(\pi^{-}, d\right) X$ and ${ }^{11} \mathrm{~B}\left(\pi^{-}, d d\right) X$ reactions. The boron data were published in [2], but the proposed novel approach to study the configuration of

\footnotetext{
1) e-mail: chernyshev@mephi.ru
}

the ground state of ${ }^{7} \mathrm{He}$ is used here for the first time. Also, the results of the measurements on beryllium targets are new.

The measurements were carried out on a low-energy pion beam from the LANL accelerator in Los Alamos using a two-arm multilayer semiconductor spectrometer [3]. A negative pion beam with the energy of $30 \mathrm{MeV}$ passed through a moderator and was stopped in a thin $\left(\sim 25 \mu \mathrm{g} / \mathrm{cm}^{2}\right)$ target. The secondary charged particles, produced upon absorption of the stopped pions, were detected by two semiconductor telescopes with silicon detectors. The achieved energy resolution for singlecharged particles throughout the entire energy range was $\sim 0.45 \mathrm{MeV}$ (FWHM). The resolution for the detection of pairs of single-charged particles using the missing mass $(M M)$ method amounts to $\sim 1 \mathrm{MeV}$. In the measurements the error in the absolute energy calibration did not exceed $0.1 \mathrm{MeV}$.

The missing mass spectrum obtained in inclusive measurements of the ${ }^{9} \operatorname{Be}\left(\pi^{-}, d\right) X$ reaction is shown in Fig. 1. The experimental spectrum was treated as a sum of Breit-Wigner distributions and $N$-particle $(N>2)$ distributions over the phase volumes. The least squares method was used to reveal the ${ }^{7} \mathrm{He}$ states. A satisfactory description could be achieved by introducing three ${ }^{7} \mathrm{He}$ states and four distributions over the phase volumes.

The ground state and the two excited states of ${ }^{7} \mathrm{He}$ appear as discrete peaks in Fig. 1. The intense continuous spectrum is well reproduced throughout the wide energy interval by the phase volumes of the $\pi^{-}+{ }^{9} \mathrm{Be} \longrightarrow$ $\longrightarrow d+{ }^{6} \mathrm{He}+n$, and $\pi^{-}+{ }^{9} \mathrm{Be} \longrightarrow d+{ }^{6} \mathrm{He}^{*}(1.8 \mathrm{MeV})+n$ reactions (curves 2 and 3 in Fig. 1 ). The multibody dis- 


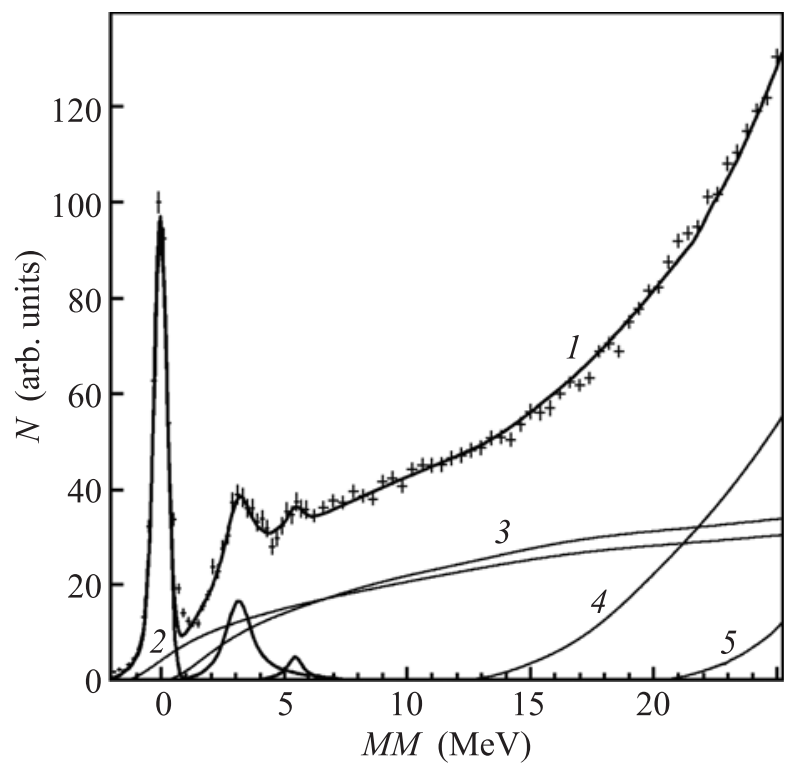

Fig. 1. The $M M$ spectrum of the reaction $\pi^{-}+{ }^{9} \mathrm{Be} \longrightarrow$ $d+X$. Points with error bars denote the experimental data. Curve 1-summary spectrum; peaks are the Breit-Wigner distributions for the ground and excited states; distributions over phase volumes: $2-\pi^{-}+{ }^{9} \mathrm{Be} \longrightarrow d+{ }^{6} \mathrm{He}+n$, $3-\pi^{-}+{ }^{9} \mathrm{Be} \longrightarrow d+{ }^{6} \mathrm{He}^{*}(1.8 \mathrm{MeV})+n, 4-\pi^{-}+{ }^{9} \mathrm{Be} \longrightarrow$ $d+{ }^{5} \mathrm{He}+2 n, 5-\pi^{-}+{ }^{9} \mathrm{Be} \longrightarrow d+{ }^{4} \mathrm{He}+3 n$

tributions 4 and 5 start contributing only at higher $M M$ energies.

The missing mass spectrum obtained on correlation measurements of the ${ }^{11} \mathrm{~B}\left(\pi^{-}, d d\right) X$ reaction is shown in [2]. As in the case of Fig. 1, the intense continuous spectrum is well reproduced by the phase volumes of the $\pi^{-}+{ }^{11} \mathrm{~B} \longrightarrow d+d+{ }^{6} \mathrm{He}_{\text {g.s. }}+n, \pi^{-}+{ }^{11} \mathrm{~B} \longrightarrow d+d+$ ${ }^{6} \mathrm{He}^{*}(1.8 \mathrm{MeV})+n$ and $\pi^{-}+{ }^{11} \mathrm{~B} \longrightarrow d+d+{ }^{5} \mathrm{He}+2 n$ reactions.

One of the common features of the spectra is the dominant contribution to the continuous spectrum from the final states with the formation of the ground and the first excited state of ${ }^{6} \mathrm{He}$. The other common feature is the absence of a significant contribution to the spectra from the channels with three nucleons in the final state: $\pi^{-}+{ }^{9} \mathrm{Be} \longrightarrow d+{ }^{4} \mathrm{He}+3 n$ and $\pi^{-}+{ }^{11} \mathrm{~B} \longrightarrow$ $d+d+{ }^{4} \mathrm{He}+3 n$. This observation constitutes one of the most important outcomes of this work. It implies that, in the threshold region, a three-neutron system can be formed only through the creation of the first excited state of ${ }^{6} \mathrm{He}^{*}$ and its subsequent decay. This means that the probability to create a halo with three non-interacting neutrons ("true" halo) is very small. The observation of this effect in two different reactions provides grounds to the conclusion that the ${ }^{4} \mathrm{He}+3 n$ structure is not manifested in the ground state of ${ }^{7} \mathrm{He}$. This hypothesis has never been proposed before.

The main configurations reproducing the sum of partial phase volumes in the near-threshold region are $d+{ }^{6} \mathrm{He}+n$, and $d+{ }^{6} \mathrm{He}^{*}(1.8 \mathrm{MeV})+n$ for the reaction ${ }^{9} \mathrm{Be}\left(\pi^{-}, d\right) X$, and $d+d+{ }^{6} \mathrm{He}+n$, and $d+d+$ ${ }^{6} \mathrm{He}^{*}(1.8 \mathrm{MeV})+n$ for the reaction ${ }^{11} \mathrm{~B}\left(\pi^{-}, d d\right) X$. This observation hints of a possible existence of a complicated "halo-like" configuration in ${ }^{7} \mathrm{He}$ with all three neutrons located outside of the alpha particle core. The structure of this complicated halo-like state would be determined by correlations of neutrons in the $p_{3 / 2}$ and $p_{1 / 2}$ shells. This result would be in agreement with the previous works $[4,5]$ pointing out to a considerable mixing of configurations containing neutrons in the above-mentioned states.

To confirm the existence of the halo-like structure in ${ }^{7} \mathrm{He}$, it would be necessary to estimate the radius of this nucleus. Having this goal in mind, as the next step we intend to analyze the existing data on the $\left(t,{ }^{3} \mathrm{He}\right)$ charge-exchange reactions on ${ }^{6} \mathrm{Li}$ and ${ }^{7} \mathrm{Li}$.

Full text of the paper is published in JETP Letters journal. DOI:

1. I. Tanihata, H. Hamagaki, O. Hashimoto, Y. Shida, N. Yoshikawa, K. Sugimoto, O. Yamakawa, T. Kobayashi, and N. Takahashi, 55, 2676 (1985).

2. Yu. B. Gurov, L. Yu. Korotkova, S. V. Lapushkin, R. V. Pritula, V. G. Sandukovsky, M. V. Tel'kushev, and B. A. Chernyshev, JETP Lett. 101, 69 (2015).

3. Yu. B. Gurov, S. V. Lapushkin, B. A. Chernyshev, and V. G. Sandukovsky, Phys. Part. Nucl. 40, 558 (2009).

4. I. Tanihata, H. Savajols, and R. Kanungo, Progr. Part. Nucl. Phys. 68, 215 (2013).

5. Yu. Aksyutina, H. T. Johansson, T. Aumann et al. (Collaboration), Phys. Lett. B 679, 191 (2009). 